\title{
Study on the low mass Drell-Yan production at the CERN LHC within the dipole formalism
}

\author{
M. B. Gay Ducati, M. T. Griep, and M. V. T. Machado \\ High Energy Physics Phenomenology Group, GFPAE IF-UFRGS Caixa Postal 15051, CEP 91501-970, \\ Porto Alegre, Rio Grande do Sul, Brazil
}

(Received 26 July 2013; revised manuscript received 14 January 2014; published 19 February 2014)

\begin{abstract}
We present results for the low-mass Drell-Yan (DY) production in proton-proton collisions at the LHC in the color dipole formalism. The DY differential cross sections at $\sqrt{s}=7 \mathrm{TeV}$ as a function of dilepton rapidity, transverse momentum, and invariant mass are discussed. We impose kinematical cuts related to the low-mass DY production investigated by the ATLAS and LHCb Collaborations.
\end{abstract}

DOI: 10.1103/PhysRevD.89.034022

PACS numbers: 12.38.Bx, 13.60.Hb, 13.85.Lg

\section{INTRODUCTION}

The cross sections for producing lepton pairs by the Drell-Yan (DY) process have been proven to still fulfill the factorization property and are finite to first order in perturbation theory at sufficiently large transverse momenta, $p_{T}$. On the other hand, there is an extensive program of research treating the low- $p_{T}$ region as the conventional factorization approaches give divergent results at $p_{T} \rightarrow 0$ (see Ref. [1] and references therein). In particular, in Ref. [2] it was shown that the differential cross section in the region $p_{T} \geq M_{\ell \ell} / 2$ is driven by subprocesses initiated by incident gluons and therefore massive lepton-pair differential cross sections are useful sources of constraints on the gluon density. That study, which used next-to-leading-order QCD and $p_{T}$ resummation, was generalized in Ref. [3] to polarized scattering and in Ref. [4] to electroweak gauge boson production. The study of the DY cross section with dileptons carrying large values of transverse momentum has a long history and it is related to deep inelastic lepton scattering (DIS), prompt photon production, and jet production as an important probe of short-distance hadron dynamics. Besides helping to constrain the parton distribution functions (PDFs) in the nucleons, the great appeal of the production of dileptons in the DY process in nuclear targets is that they are colorless probes of the dynamics of quarks and gluons [5]. Namely, they escape unscathed through the colored medium of the high-energy collision. Thus, the dileptons can be a powerful probe of the initial state of matter created in heavy-ion collisions, since they interact with the medium only electromagnetically and therefore provide a baseline for the interpretation of jet-quenching models. Along these lines, it has been shown $[6,7]$ that these electromagnetic probes are crucial to determine the dominant physics in the forward region at the Relativistic Heavy Ion Collider (RHIC) and at the LHC.

It has been shown [8] that both direct (prompt) photon production and Drell-Yan dilepton pair production processes can be described within the same color dipole approach without any free parameters. Such a formalism, developed in Ref. [9] for the case of the total and diffractive cross sections, can be also applied to radiation [10,11]. In the rest frame of a target, the DY process looks like a bremsstrahlung [12] of a massive photon from an incoming quark. The photons can be emitted before or after a quark is scattered on a proton. Although in the process of electromagnetic bremsstrahlung by a quark no real quark dipole participates, the cross section can be expressed through the more elementary cross section $\sigma_{\text {dip }}$ of the interaction of a $Q \bar{Q}$ dipole [12]. The relation between this formalism and the usual collinear perturbative QCD (pQCD) factorization has been studied in detail in Ref. [13]. The dipole formalism offers an easy way to calculate the transversemomentum distribution in the DY process even in the low- $p_{T}$ region. The corresponding phenomenology investigating the role played by high-energy approaches in the DY $p_{T}$ distribution has been investigated in Ref. [14]. Results from order $\alpha_{s}$ in the parton model cannot be directly compared to such an approach since it is not an expansion in any parameter. All contributions from higher-order graphs enhanced by a factor $\ln \left(1 / x_{2}\right)$ and even nonperturbative corrections are included. For instance, in Ref. [15] a twist expansion in powers of $\left(\frac{Q_{\text {sat }}}{M_{\ell \ell}}\right)^{2}$ ( $Q_{\text {sat }}$ is the saturation scale and $M_{\ell \ell}$ is the invariant dilepton mass) was developed. It was shown that the leading twist is a good approximation to the full result for masses $M_{\ell \ell} \geq 6 \mathrm{GeV}$. Recently, the diffractive DY cross section has been investigated in detail within the dipole framework [16]. The corresponding factorizationbreaking effects in diffractive DY lead to very distinct properties of the observables compared to QCD factorization-based calculations.

Our goal is to investigate in detail the low-mass DY cross section at the LHC energies using the color dipole approach, and to discuss several phenomenological aspects. We focus mainly on forward rapidities at the energy available at the LHC. The paper is organized as follows. In the next section we summarize the main formula for the DY differential cross section within the dipole framework, 
which is suitable for forward rapidities and allows one to incorporate parton saturation effects. In the last section we show our numerical results and predictions and summarize our main conclusions.

\section{THEORETICAL FRAMEWORK}

This section resumes the theoretical treatment for DrellYan production in $p p$ collisions in high energies considered in our study. We will use the dipole approach, which is well suited for high-energy processes, i.e., small parton momentum fraction in the target $x_{2} \propto M_{\ell \ell} / \sqrt{s}$, and its range of validity is expected to be near $x_{2}<0.01$. The lowmass DY production surely probes the small- $x$ physics, especially the forward-rapidity case. One advantage of such an approach is the ability to describe simultaneously the direct photon and dilepton production in the same theoretical framework, and it provides finite cross sections in the limit $p_{T} \rightarrow 0$. The transverse-momentum $p_{T}$ distribution of the virtual photon bremsstrahlung in quark-nucleon interactions, integrated over the final quark transverse momentum, was derived in Ref. [12] in terms of the dipole formalism,

$$
\begin{aligned}
\frac{d^{3} \sigma^{q N}\left(q \rightarrow q \gamma^{*}\right)}{d(\ln \alpha) d^{2} \vec{p}_{T}}= & \frac{1}{(2 \pi)^{2}} \sum_{\mathrm{in}, f} \sum_{L, T} \int d^{2} \vec{r}_{1} d^{2} \vec{r}_{2} e^{i \vec{p}_{T} \cdot\left(\vec{r}_{1}-\vec{r}_{2}\right)} \\
& \times \phi_{\gamma q}^{\star T, L}\left(\alpha, \vec{r}_{1}\right) \phi_{\gamma q}^{T, L}\left(\alpha, \vec{r}_{2}\right) \\
& \times\left[\frac{1}{2}\left(\sigma_{\mathrm{dip}}\left(x_{2}, \alpha r_{1}\right)+\sigma_{\mathrm{dip}}\left(x_{2}, \alpha r_{2}\right)\right)\right. \\
& \left.-\frac{1}{2} \sigma_{\mathrm{dip}}\left(x_{2}, \alpha\left(\vec{r}_{1}-\vec{r}_{2}\right)\right)\right]
\end{aligned}
$$

where $\vec{r}_{1}$ and $\vec{r}_{2}$ are the quark-photon transverse separations in the two radiation amplitudes contributing to the cross section, $\sigma_{\text {dip }}$. The parameter $\alpha$ is the relative fraction of the quark momentum carried by the photon, and is the same in both amplitudes, since the interaction does not change the sharing of longitudinal momentum. In the equation above, $T$ stands for transverse and $L$ for longitudinal photons. The energy dependence of the dipole cross section, which comes through the variable $x_{2}=2\left(p_{1} \cdot q\right) / s=\left(M_{T} / \sqrt{s}\right) e^{-y}$ (with $\left.M_{T}=\sqrt{M^{2}+p_{T}^{2}}\right)$, where $p_{1}$ is the projectile fourmomentum and $q$ is the four-momentum of the dilepton, is generated by the additional radiation of gluons which can be resummed in the leading $\ln (1 / x)$ approximation.

In Eq. (1) the light-cone wave function of the projectile quark $\gamma q$ fluctuation has been decomposed into transverse $\phi_{\gamma q}^{T}(\alpha, \vec{r})$ and longitudinal $\phi_{\gamma q}^{L}(\alpha, \vec{r})$ components, and an average over the initial quark polarization and a sum over all final polarization states of the quark and photon is performed. The expressions for the $T$ and $L$ wave-function components are well known at the lowest order $[12,14]$. The hadron cross section can be obtained from the elementary partonic cross section (1) by summing up the contributions from quarks and antiquarks weighted with the corresponding PDFs,

$$
\begin{aligned}
\frac{d^{4} \sigma\left(p p \rightarrow \ell^{+} \ell^{-} X\right)}{d y d M^{2} d^{2} \vec{p}_{T}}= & K_{\mathrm{eff}} \frac{\alpha_{\mathrm{em}}}{3 \pi M^{2}} \int_{x_{1}}^{1} \frac{d \alpha}{\alpha} F_{2}^{p}\left(\frac{x_{1}}{\alpha}, \mu^{2}\right) \\
& \times \frac{d \sigma^{q N}\left(q \rightarrow q \gamma^{*}\right)}{d(\ln \alpha) d^{2} \vec{p}_{T}},
\end{aligned}
$$

where the PDFs of the projectile have entered in a combination which can be written in terms of the proton structure function $F_{2}^{p}$, with $x_{1}=\left(M_{T} / \sqrt{s}\right) e^{y}$. For the hard scale $\mu$ entering into the proton structure function in Eq. (2) we take $\mu^{2}=\beta\left[\left(1-x_{1}\right) M^{2}+p_{T}^{2}\right]$, and the energy scale of the dipole cross section in Eq. (1) is given by the variable $x_{2}$. The dependence of the cross section on the choice for the hard scale can be obtained by varying the $\beta$ value (the default value here is $\beta=1$ ). The quantity $K_{\text {eff }}$ takes into account the effective higher-order DY contributions. For simplicity we take the expression [17]

$$
K_{\mathrm{eff}}\left(\mu^{2}\right)=1+\frac{\alpha_{s}\left(\mu^{2}\right)}{2 \pi}\left(1+\frac{4}{3} \pi^{2}\right),
$$

where the running coupling constant is computed at the scale $\mu^{2}=M^{2}$.

An important piece in the color dipole calculations is the dipole cross section. It is theoretically unknown, although several parametrizations have been proposed. Here, we consider some analytical parametrizations which rely on the geometric scaling property. In this case, they are a function of a scaling variable $r Q_{\text {sat }}(x)$, where $Q_{\text {sat }}$ is the socalled saturation scale. It defines the transverse-momentum scale where parton recombination physics is relevant and in general is modeled as $Q_{\text {sat }} \propto x^{-\lambda / 2}$. A common feature of these models is that for decreasing $x$, the dipole cross section saturates for smaller dipole sizes. In addition, at small $r$, as perturbative QCD implies $\sigma \sim r^{2}$, they vanish, i.e., the color transparency phenomenon occurs. In a general form, they can be parametrized as [18]

$$
\sigma_{\text {dip }}(x, \vec{r} ; \gamma)=\sigma_{0}\left[1-\exp \left(-\frac{r^{2} Q_{\text {sat }}^{2}}{4}\right)^{\gamma_{\text {effi }}}\right] \text {, }
$$

where the quantity $\gamma_{\text {eff }}$ is the effective anomalous dimension. The Golec-Biernat-Wusthoff (GBW) parametrization [18] uses $\gamma_{\text {eff }}=1$ and the remaining parameters are fitted to DIS HERA data at small $x$. The saturation scale is defined as $Q_{\text {sat }}^{2}(x)=\left(\frac{x_{0}}{x}\right)^{\lambda}$. This parametrization gives a rather good description of DIS data at $x<10^{-2}$.

The main difference among the distinct phenomenological models using parametrizations like Eq. (4) comes from the predicted behavior for the anomalous dimension, which determines the transition from the nonlinear to the extended geometric scaling regime, as well as from the extended 
geometric scaling to the Dokshitzer-Gribov-LipatovAltarelli-Parisi (DGLAP) regime. It is the behavior of $\gamma$ that determines the diminishing of the hadronic cross section as $p_{T}$ increases. Several models in the literature have considered the general form $\gamma_{\mathrm{eff}}=\gamma_{\mathrm{sat}}+\Delta\left(x, r ; p_{T}\right)$, where $\gamma_{\text {sat }}$ is the anomalous dimension at the saturation scale and $\Delta$ mimics the onset of the geometric scaling region and DGLAP regime. In order to take this possibility into account, here we also will consider the phenomenological saturation model proposed in Ref. [19] which encodes the main properties of the saturation approaches, with the dipole cross section parametrized as follows:

$$
\sigma_{\text {dip }}(x, r)=\sigma_{0} \begin{cases}\mathcal{N}_{0}\left(\frac{\bar{\tau}^{2}}{4}\right)^{\gamma_{\text {eff }}(x, r),}, & \text { for } \bar{\tau} \leq 2, \\ 1-\exp \left[-a \ln ^{2}(b \bar{\tau})\right], & \text { for } \bar{\tau}>2,\end{cases}
$$

where $\bar{\tau}=r Q_{\text {sat }}(x)$ and the expression for $\bar{\tau}>2$ (saturation region) has the correct functional form, as obtained from the theory of the color glass condensate (CGC) [19]. For the color transparency region near the saturation border $(\bar{\tau} \leq 2)$, the behavior is driven by the effective anomalous dimension $\gamma_{\text {eff }}(x, r)=\gamma_{\text {sat }}+\frac{\ln (2 / \tilde{\tau})}{\kappa \lambda y}$, where $\gamma_{\text {sat }}=0.63$ is the leading-order (LO) Balitsky-Fadin-Kuraev-Lipatov anomalous dimension at the saturation limit.

Before computing numerically the cross section given by Eq. (2), we discuss the semianalytical calculation which is allowed in the color dipole picture in the color transparency region. In that case, an expression for the $p_{T}$ distribution can be written using Eq. (2) and the expressions for the transverse-momentum $p_{T}$ distribution of photon bremsstrahlung in quark-nucleon interactions [Eq. (1)]. The explicit equation for the DY differential cross section, Eq. (2), reads

$$
\begin{aligned}
& \frac{d^{4} \sigma\left(p p \rightarrow \ell^{+} \ell^{-} X\right)}{d y d M^{2} d^{2} \vec{p}_{T}} \\
& =\frac{\alpha_{\mathrm{em}}^{2}}{6 \pi^{3} M^{2}} \int_{x_{1}}^{1} \frac{d \alpha}{\alpha} F_{2}^{p}\left(\frac{x_{1}}{\alpha}, Q^{2}=\mu^{2}\right) \\
& \quad \times\left\{\left[m_{q}^{2} \alpha^{4}+2 M^{2}(1-\alpha)^{2}\right]\left[\frac{\mathcal{I}_{1}}{\left(p_{T}^{2}+\varepsilon^{2}\right)}-\frac{\mathcal{I}_{2}}{4 \varepsilon}\right]\right. \\
& \left.\quad+\left[1+(1-\alpha)^{2}\right]\left[\frac{\varepsilon p_{T} \mathcal{I}_{3}}{\left(p_{T}^{2}+\varepsilon^{2}\right)}-\frac{\mathcal{I}_{1}}{2}+\frac{\varepsilon \mathcal{I}_{2}}{4}\right]\right\},
\end{aligned}
$$

where $\varepsilon^{2}=(1-\alpha) M^{2}+\alpha^{2} m_{q}^{2}$. The quantities $\mathcal{I}_{1,2,3}$ are Hankel's integral transforms of order $0\left(\mathcal{I}_{1,2}\right)$ and order 1 $\left(\mathcal{I}_{3}\right)$, given by

$$
\begin{aligned}
& \mathcal{I}_{1}=\int_{0}^{\infty} d r r J_{0}\left(p_{T} r\right) K_{0}(\varepsilon r) \sigma_{\mathrm{dip}}\left(x_{2}, \alpha r\right), \\
& \mathcal{I}_{2}=\int_{0}^{\infty} d r r^{2} J_{0}\left(p_{T} r\right) K_{1}(\varepsilon r) \sigma_{\mathrm{dip}}\left(x_{2}, \alpha r\right), \\
& \mathcal{I}_{3}=\int_{0}^{\infty} d r r J_{1}\left(p_{T} r\right) K_{1}(\varepsilon r) \sigma_{\mathrm{dip}}\left(x_{2}, \alpha r\right) .
\end{aligned}
$$

Here, $K_{0,1}(x)$ denotes the modified Bessel function of the second kind and $m_{q}$ is an effective quark mass.

Considering a kinematic interval where the dipole cross section is dominated by the color transparency region, one can use the GBW parametrization and take its small- $r$ limit to analytically compute the integrals in Eq. (6). In this case, we can take the approximation $\sigma_{\text {dip }} \approx \sigma_{0}\left(r^{2} Q_{\text {sat }}^{2}\right)$ in the region where $p_{T} \gg Q_{\text {sat }}$. At the LHC energies, the typical saturation scale is in units of $\mathrm{GeV}$. For instance, at midrapidity $x_{2} \simeq M / \sqrt{s}$, which gives for $\langle M\rangle=10 \mathrm{GeV}$ a value $x_{2} \approx 10^{-3}$ at $7 \mathrm{TeV}$. Then, the saturation scale is of order $\quad Q_{\text {sat }}=\left(x_{0} / x_{2}\right)^{\lambda / 2} \mathrm{GeV} \approx 0.8 \mathrm{GeV} \quad$ (with $x_{0}=$ $3.04 \times 10^{-4}$ and $\left.\lambda=0.288\right)$. The final results for the hadron cross section then are

$$
\begin{aligned}
& \frac{d \sigma\left(p p \rightarrow \ell^{+} \ell^{-} X\right)}{d y d M^{2} d^{2} \vec{p}_{T}} \\
& \approx \frac{\alpha_{\mathrm{em}}^{2} \sigma_{0} Q_{\mathrm{sat}}^{2}}{6 \pi^{3} M^{2}} \int_{x_{1}}^{1} \frac{d \alpha}{\alpha} F_{2}^{p}\left(\frac{x_{1}}{\alpha}, Q^{2}\right) \\
& \quad \times\left\{\left[m_{q}^{2} \alpha^{4}+2 M^{2}(1-\alpha)^{2}\right]\left[\frac{p_{T}^{2}}{\left(p_{T}^{2}+\varepsilon^{2}\right)^{4}}\right]\right. \\
& \left.\quad+\left[1+(1-\alpha)^{2}\right]\left[\frac{p_{T}^{4}+\varepsilon^{4}}{2\left(p_{T}^{2}+\varepsilon^{2}\right)^{4}}\right]\right\} .
\end{aligned}
$$

In what follows we compute the differential cross sections of low-mass DY production in hadron-hadron collisions, focusing on the recent measurements done by the ATLAS [20] and LHCb [21] Collaborations at the LHC.

\section{NUMERICAL RESULTS}

Let us now present some numerical calculations concerning the low-mass dilepton production in the LHC energy regime of $\sqrt{s}=7 \mathrm{TeV}$. For the proton structure function in Eq. (2) we have taken the Abramowicz-LevinLevy-Maor parametrization [22], which is valid in the kinematic range we are interested in. The sensitivity to a different choice for $F_{2}$ is very small. Moreover, in order to account for the threshold region $x_{2} \rightarrow 1$, we have corrected the dipole cross section by multiplying it by a threshold factor $\left(1-x_{2}\right)^{7}$. We consider $m_{q}=0.2 \mathrm{GeV}$ for the effective quark mass. In Fig. 1, we show the results for the differential cross section, $d^{3} \sigma / d M^{2} d Y d p_{T}$ (in units of $\mathrm{pb})$, as a function of the dilepton transverse momentum $p_{T}$. Here, the predictions are obtained using the GBW dipole cross section (bold curves) and the CGC dipole cross section (thin curves) and using the hard scale $\mu^{2}=\left(1-x_{1}\right) M^{2}+p_{T}^{2}$. Notice that the $p_{T}$ spectrum is quite sensitive to the particular model of dipole cross section (especially at large transverse momentum) as it depends on the behavior of the effective anomalous dimension, as discussed in the previous section. In the left panel we show the case for a fixed invariant mass $M=$ $6 \mathrm{GeV}$ and for a few sample values of dilepton rapidity 

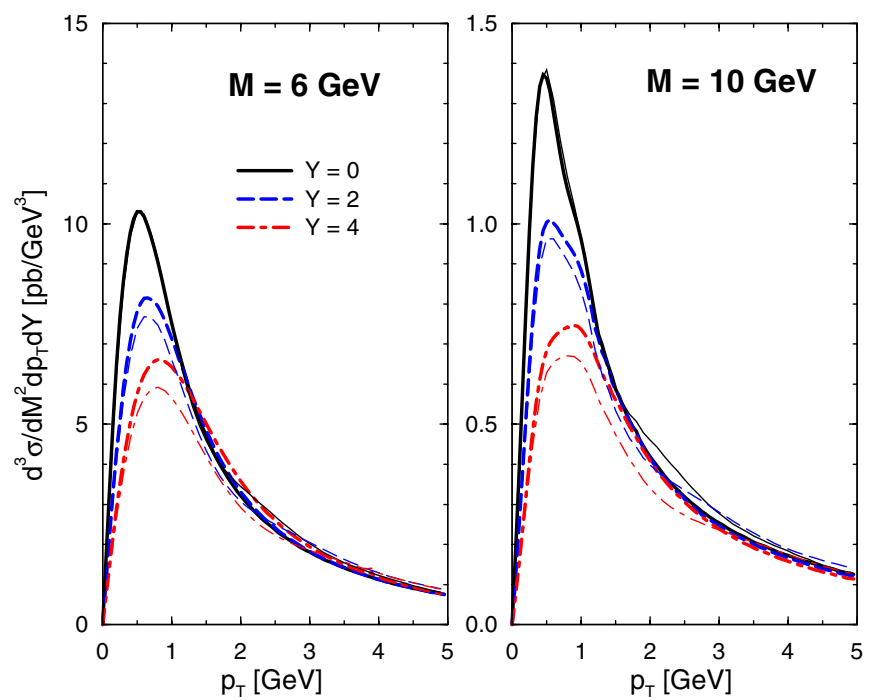

FIG. 1 (color online). Low-mass DY differential cross sections, $d^{3} \sigma / d M^{2} d Y d p_{T}$, as a function of dilepton transverse momentum, $p_{T}$, at an energy of $\sqrt{s}=7 \mathrm{TeV}$. The plots are shown for fixed dilepton mass $(M=6$ and $10 \mathrm{GeV})$ and distinct lepton pair rapidities $(Y=0,2,4)$. The results are presented using the GBW dipole cross section (bold curves) and the CGC dipole cross section (thin curves).

including central and forward rapidities, i.e., $Y=0,2$ and 4 , respectively. The same notation holds for the right panel, where now the invariant mass is $M=10 \mathrm{GeV}$. As expected, the large-rapidity cases give smaller cross sections and the peak in the distributions is shifted to larger values of the transverse momentum. In the kinematical situation investigated here the peak is located at a momentum around $p_{T} \approx 1 \mathrm{GeV}$. The shift and location of the peak can be understood by looking at the semianalytical expression, Eq. (7). A clear problem in the numerical calculations using the full $p_{T}$ spectrum is due to the strongly oscillating integrand appearing in Eq. (6), which makes the $p_{T}$ integrations a delicate task mainly for high mass values.

In Fig. 2 we show the invariant mass distribution at midrapidities obtained from two different implementations of the dipole cross section taken from recent phenomenological works. We considered the GBW model (dot-dashed line) and the phenomenological saturation model, labeled here CGC (solid line), which involves a running anomalous dimension. The main deviation between these two models occurs at large $p_{T}$, which gives distinct overall normalizations for the dilepton invariant mass distribution. The considered cuts are along the lines presented by the ATLAS analysis [20] for the low-mass Drell-Yan dimuon process. The selection cuts in that analysis at an energy of $\sqrt{s}=7 \mathrm{TeV}$ and integrated luminosity of $36 \mathrm{pb}^{-1}$ were low muon transverse momentum, $p_{T}^{\mu}>6 \mathrm{GeV}$, and low dimuon mass region, $12<M_{\ell \ell}<66 \mathrm{GeV}$. Here, we consider the integration over the boson rapidity in the range $\left|Y_{\ell \ell}\right|<2.5$ and dilepton transverse momentum

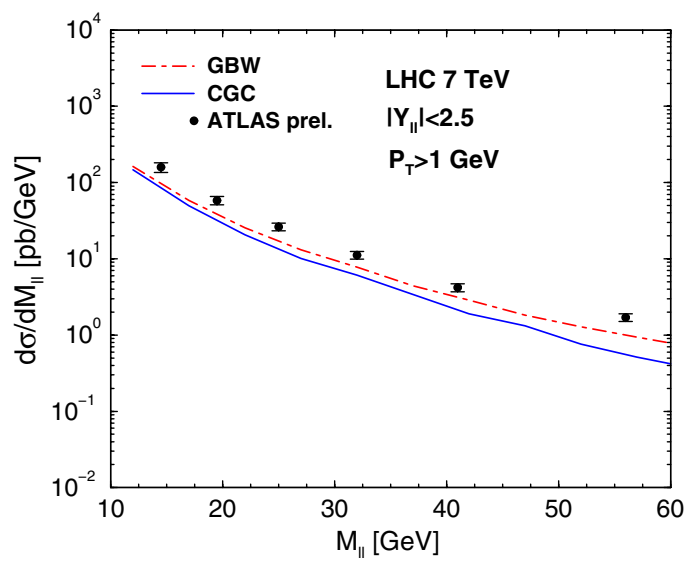

FIG. 2 (color online). Invariant mass distribution in the range $12<M_{\ell \ell}<60 \mathrm{GeV}$. The imposed cuts at an energy of $\sqrt{s}=$ $7 \mathrm{TeV}$ are lepton pair rapidities $\left|Y_{\ell \ell}\right|<2.5$ and the dilepton transverse momentum $p_{T} \geq 1 \mathrm{GeV}$. Preliminary ATLAS data [20] are shown for sake of comparison.

$p_{T} \geq 1 \mathrm{GeV}$. Distinct $p_{T}$ cuts will lead to a different overall normalization for the invariant mass distribution. At this stage we did not impose the selected cuts on individual muons, as was done in the ATLAS analysis. Two different studies were done by ATLAS [20]: one with a selection of muons of different minimum $p_{T}^{\mu}$ and no requirement on boson selection except for the invariant mass constraint (asymmetric analysis), and one with a selection of minimum $p_{T}^{\mu}$ for muons and where a requirement on the rapidity of the boson is applied (symmetric analysis). The results presented here are somewhat consistent with the extrapolated Born-level differential cross section using the symmetric analysis. For sake of comparison, we include the preliminary data [20] in Fig. 2 (filled circles).

The main $M^{2}$ dependence of the DY cross section can be quantitatively understood in the color dipole framework. In the case of the integrated cross section on $p_{T}$, it was shown in Ref. [15] that a twist expansion in positive powers of the ratio $\left(\frac{Q_{\text {sat }}}{M_{\ell \ell}}\right)^{2}$ can be performed. The leading-twist contribution reads as [15]

$$
\begin{aligned}
\frac{d^{2} \sigma}{d y d M^{2}} \approx & \frac{\alpha_{\mathrm{em}}^{2} \sigma_{0}}{12 \pi^{2} M^{2}} \frac{Q_{\mathrm{sat}}^{2}\left(x_{2}\right)}{M^{2}} F_{2}^{p}\left(x_{1}, M^{2}\right) \\
& \times\left\{\frac{4}{3} \gamma_{E}-1+\frac{2}{3}\left[\psi\left(\frac{5}{2}\right)-\ln \frac{Q_{\mathrm{sat}}^{2}\left(x_{2}\right)}{4 M^{2}\left(1-x_{1}\right)}\right]\right\},
\end{aligned}
$$

modulo contributions of $\mathcal{O}\left(1-x_{1}\right)$. The exact leadingtwist formula is quite close to the all-twist result at the LHC energies in the region of $M>6 \mathrm{GeV}$ [15]. In the central rapidities considered above, $\langle Y\rangle=0$, the saturation scale changes from $\left\langle Q_{\text {sat }}^{2}\right\rangle \simeq 0.4 \mathrm{GeV}^{2}$ down to $\left\langle Q_{\text {sat }}^{2}\right\rangle \simeq 0.2 \mathrm{GeV}^{2}$ in the invariant mass range $12<M_{\ell \ell}<66 \mathrm{GeV}$, respectively. In this situation, the analytical result given in Eq. (7) 

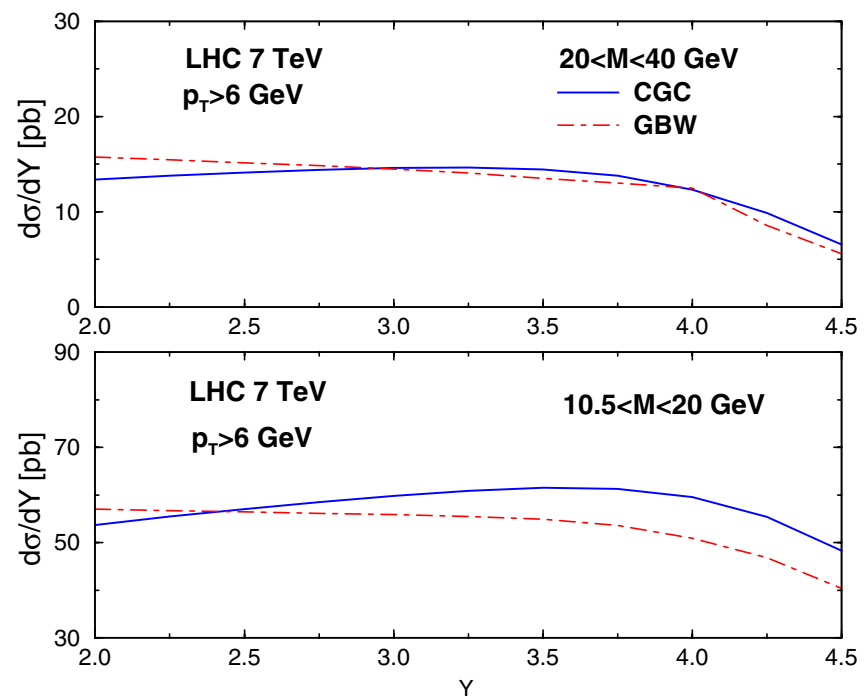

FIG. 3 (color online). The dilepton rapidity distribution at $\sqrt{s}=7 \mathrm{TeV}$ imposing the cut on the dimuon transverse momentum $p_{T}>6 \mathrm{GeV}$ and two invariant mass regions: (upper plot) $20 \leq M_{\ell \ell} \leq 40 \mathrm{GeV}$ and (lower plot) $10.5 \leq M_{\ell \ell} \leq 20 \mathrm{GeV}$.

should be quite reliable. It would be timely in the future to investigate the effects of QCD DGLAP evolution in the dipole cross section and also the impact-parameter dependence in the CGC model, as was done recently in Refs. [23] and [24].

Now, we focus on the forward rapidity region. In Fig. 3, the rapidity distribution, $d \sigma / d Y$, is computed for the interval $2<Y<4.5$ considering the two phenomenological models (same notation as the previous plot) and using the hard scale $\mu^{2}=\frac{1}{2}\left[\left(1-x_{1}\right) M^{2}+p_{T}^{2}\right]$. It was found that the rapidity distribution is sensitive to the chosen hard scale, which also occurs for the LO pQCD approach. Here, the deviations between the models are stronger than for the mass distribution. The main point is that the rapidity distribution is driven by the effective anomalous dimension, which is distinct in the models. We have imposed a cut for the dilepton transverse momentum of $p_{T}>6 \mathrm{GeV}$ and two distinct intervals of invariant mass. Namely, in the upper plot one has $20 \leq M_{\ell \ell} \leq 40 \mathrm{GeV}$ whereas in the lower plot one has $10.5 \leq M_{\ell \ell} \leq 20 \mathrm{GeV}$. The motivation for such a cut is due to the recent LHCb Collaboration [21] measurement of the low-mass DY cross section. The measurements collected with an integrated luminosity of $37 \mathrm{pb}^{-1}$ are for the dimuon final state having muons within pseudorapidities of 2 to 4.5 , and muon transverse momentum $p_{T}^{\mu}>3 \mathrm{GeV}\left(p_{T}^{\mu}>15 \mathrm{GeV}\right.$ for higher masses) in two distinct mass regions. In the forward rapidities considered here, the saturation scale is in the interval $0.6 \leq\left\langle Q_{\text {sat }}^{2}\right\rangle \leq 1.2 \mathrm{GeV}^{2}$ for $\left\langle M_{\ell \ell}\right\rangle \simeq$ $15.25 \mathrm{GeV}$. Slightly lower values are found also for higher masses, $\left\langle M_{\ell \ell}\right\rangle \simeq 30 \mathrm{GeV}$.

Finally, we check the energy dependence of the DY differential cross section within the color dipole picture.
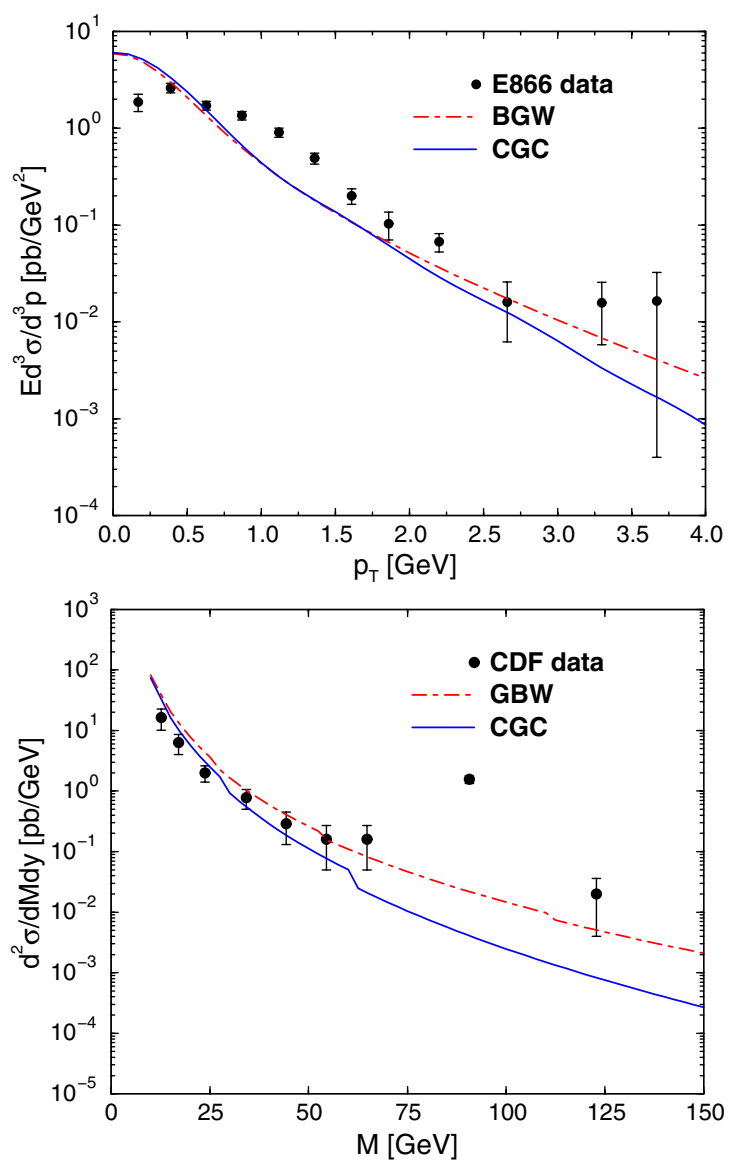

FIG. 4 (color online). The DY invariant cross section (upper panel) at $\sqrt{s}=39 \mathrm{GeV}$ as a function of $p_{T}$ compared to the E866 Collaboration data [25]. In the bottom panel, the differential cross section $d^{2} \sigma / d M d y$ at $\sqrt{s}=1800 \mathrm{GeV}$ as a function of invariant mass is presented and compared to the CDF Collaboration data [26].

In Fig. 4 (upper panel) we show the invariant cross section as a function of $p_{T}$ at energy $\sqrt{s}=39 \mathrm{GeV}$. The experimental results from the E866 Collaboration [25] are also presented $\left(\left\langle x_{F}\right\rangle \simeq 0.63\right.$ and $\left.4.2 \leq M_{\mu^{+} \mu^{-}} \leq 5.2 \mathrm{GeV}\right)$. In the bottom panel, the differential cross section $d^{2} \sigma / d M d y$ (for $|y|<1$ ) is shown for the energy $\sqrt{s}=$ $1800 \mathrm{GeV}$ as a function of dilepton invariant mass. The data from the CDF Collaboration [26] are included in the plot, considering also the large invariant mass data points. The solid curves refer to CGC and dot-dashed curves to the GBW dipole cross section, respectively. The color dipole picture reasonably describes the cross section from low to high energies in the kinematical regions where it is expected to be valid. The approach is also somewhat consistent with calculations carried out in next-to-leading-order QCD at both fixed target and collider energies [2].

As a summary, we have shown that low-mass DY production can be addressed in the color dipole picture without any free parameters by using a dipole cross section 
determined from current phenomenology in DIS. It has been shown before [27] that in central rapidities at the RHIC and Tevatron saturation effects do not play a significant role for the measured range of $p_{T}$. This situation can be changed at the LHC even at midrapidities as the saturation scale is enhanced by a sizable factor. We also presented analytical results that are relevant for the calculation of the $p_{T}$ spectrum of DY dileptons.

\section{ACKNOWLEDGMENTS}

This work was partially financed by the Brazilian funding agencies CNPq and FAPERGS and by the French-Brazilian scientific cooperation project CAPES-COFECUB 744/12. M. V.T.M. thanks Murilo Rangel, Ronan McNulty, and Michael Klasen for helpful comments, and M.B.G.D. thanks M. A. Betemps for useful discussions.
[1] Z.-B. Kang, J.-W. Qiu, and W. Vogelsang, Phys. Rev. D 79, 054007 (2009).

[2] E. L. Berger, L. E. Gordon, and M. Klasen, Phys. Rev. D 58, 074012 (1998).

[3] E. L. Berger, L. E. Gordon, and M. Klasen, Phys. Rev. D 62 , 014014 (2000).

[4] M. Klasen and M. Brandt, Phys. Rev. D 88, 054002 (2013).

[5] A. L. Ayala, M. B. Gay Ducati, L. N. Epele, and C. A. Garcia Canal, Phys. Rev. C 49, 489 (1994).

[6] M. A. Betemps and M. B. Gay Ducati, Phys. Rev. D 70, 116005 (2004); Phys. Lett. B 636, 46 (2006).

[7] F. Gelis and J. Jalilian-Marian, Phys. Rev. D 76, 074015 (2007).

[8] B. Z. Kopeliovich, A. H. Rezaeian, H. J. Pirner, and I. Schmidt, Phys. Lett. B 653, 210 (2007).

[9] A. B. Zamolodchikov, B. Z. Kopeliovich, and L. I. Lapidus, Pis'ma Zh. Eksp. Teor. Fiz. 33, 612 (1981) [Sov. Phys. JETP Lett. 33, 595 (1981)].

[10] B.Z. Kopeliovich, in Proceedings of Hirschegg '95: Dynamical Properties of Hadrons in Nuclear Matter, Hirschegg January 16-21, 1995, edited by H. Feldmeyer and W. Nörenberg (GSI Publications, Darmstadt, 1995), p. 102.

[11] S. J. Brodsky, A. Hebecker, and E. Quack, Phys. Rev. D 55, 2584 (1997).

[12] B. Z. Kopeliovich, J. Raufeisen, and A. V. Tarasov, Phys. Lett. B 503, 91 (2001).

[13] J. Raufeisen, J.-C. Peng, and G. C. Nayak, Phys. Rev. D 66, 034024 (2002).

[14] M. A. Betemps, M. B. G. Ducati, M. V. T. Machado, and J. Raufeisen, Phys. Rev. D 67, 114008 (2003).
[15] K. Golec-Biernat, E. Lewandowska, and A. Stasto, Phys. Rev. D 82, 094010 (2010).

[16] R. S. Pasechnik and B. Z. Kopeliovich, Eur. Phys. J. C 71, 1827 (2011).

[17] V. Barger and R. Phillips, Collider Physics (AddisonWesley, Redwood City, CA, 1987).

[18] K. Golec-Biernat and M. Wusthoff, Phys. Rev. D 59, 014017 (1998); 60, 114023 (1999).

[19] E. Iancu, K. Itakura, and S. Munier, Phys. Lett. B 590, 199 (2004).

[20] ATLAS Collaboration, Report No. ATL-COM-PHYS2012-469 (2012).

[21] LHCb Collaboration, Report No. LHCb-CONF-2012-013 (2012).

[22] D. Gabbert and L. De Nardo, in Proceedings of the 15th International Workshop on Deep-Inelastic Scattering and Related Subjects, Munich, Germany, April 16-20, 2007, edited by G. Grindhammer and K. Sachs (DESY, Hamburg, 2007), p. 373; H. Abramowicz, E. M. Levin, A. Levy, and U. Maor, Phys. Lett. B 269, 465 (1991).

[23] A. H. Rezaeian, M. Siddikov, M. Van de Klundert, and R. Venugopalan, Phys. Rev. D 87, 034002 (2013).

[24] A. H. Rezaeian and I. Schmidt, Phys. Rev. D 88, 074016 (2013).

[25] J. C. Webb et al. (NuSea Collabroration), arXiv:hep-ex/ 0302019.

[26] F. Abe et al. (CDF Collaboration), Phys. Rev. D 49, R1 (1994).

[27] M. V. T. Machado and C. Brenner Mariotto, Eur. Phys. J. C 61, 871 (2009). 